The Higgs Puzzle: Experiment and Theory [Lepton Photon 01, Rome, July 2001]

\title{
THE HIGGS PUZZLE: EXPERIMENT AND THEORY
}

\author{
FABIO ZWIRNER \\ Dipartimento di Fisica, Università di Roma 'La Sapienza', and INFN, Sezione di Roma, \\ Piazzale Aldo Moro 2, I-00185 Roma, ITALY \\ E-mail: fabio.zwirner@roma1.infn.it
}

\begin{abstract}
The present experimental and theoretical knowledge of the physics of electroweak symmetry breaking is reviewed. Data still favor a light Higgs boson, of a kind that can be comfortably accommodated in the Standard Model or in its Minimal Supersymmetric extension, but exhibit a non-trivial structure that leaves some open questions. The available experimental information may still be reconciled with the absence of a light Higgs boson, but the price to pay looks excessive. Recent theoretical ideas, linking the weak scale with the size of possible extra spatial dimensions, are briefly mentioned. It is stressed once more that experiments at high-energy colliders, such as the Tevatron and the LHC, are the crucial tool for eventually solving the Higgs puzzle.
\end{abstract}

Rome is a city so full of religious symbols that it provides some inspiration on how to organize a talk on the physics of electroweak symmetry breaking, where firm experimental and theoretical results are mixed, so far, with a certain amount of beliefs.

\section{The Standard Model (The Orthodoxy)}

The obvious starting point for any discussion of the Higgs puzzle ( $\equiv$ 'what is the physics of electroweak symmetry breaking?') is the Standard Model (SM), by now firmly established as the renormalizable quantum field theory of strong and electroweak interactions at presently accessible energies: in the spirit of the preface, it can be called 'The Orthodoxy'. The only SM ingredient still escaping experimental detection, in a theoretical construction that works incredibly well, is the Higgs 1 boson, $H$. Its properties are controlled by some well-known parameters of the fermion and gauge sectors (including the Fermi constant $G_{F}$, which sets the value of the weak scale) plus an independent one, the Higgs mass $m_{H}$.

The elementary complex spin- 0 field $\phi$, an $S U(2)_{L}$ doublet of weak hypercharge $Y=$ $+1 / 2$ (in the normalization where $Q=T_{3 L}+$ $Y)$, is by now considered to be an essential part of what we call the SM. Indeed, it plays a fundamental role in the description of two symmetry-breaking phenomena. The first is the spontaneous breaking of the $S U(2)_{L} \times$ $U(1)_{Y}$ gauge symmetry down to the $U(1)_{Q}$ of QED, described by the following part of the SM Lagrangian:

$$
\mathcal{L}_{S}=\left(D^{\mu} \phi\right)^{\dagger}\left(D_{\mu} \phi\right)-\mu^{2} \phi^{\dagger} \phi-\lambda\left(\phi^{\dagger} \phi\right)^{2} .
$$

The second is the explicit breaking of the global flavor symmetry that is present if only gauge interactions are switched on. This is realized by the Yukawa part of the SM Lagrangian,

$$
\begin{aligned}
\mathcal{L}_{Y}= & h^{U} \overline{q_{L}} u_{R} \widetilde{\phi}+h^{D} \overline{q_{L}} d_{R} \phi \\
& +h^{E} \overline{l_{L}} e_{R} \phi+\text { h.c. },
\end{aligned}
$$

where $\widetilde{\phi}=i \sigma^{2} \phi^{*}, h^{U, D, E}$ are $3 \times 3$ complex matrices and generation indices have been omitted. Over the years, our confidence in this description has been progressively reinforced by increasingly precise tests of both these symmetry-breaking phenomena (here the focus will be on gauge symmetry breaking, since the theoretical aspects of flavor symmetry breaking are discussed in another talk at this Conference 2). However, the ultimate, crucial test of the SM remains the direct search for the Higgs particle, by far the most important experimental enterprise in today's particle physics. 


\subsection{Direct searches for the SM Higgs}

The experimental status of the searches for the SM Higgs particle is reviewed in detail in another talk at this Conference 3 . Here I will just summarize the present situation from the preliminary LEP-combined results 6 released for the conferences of Summer 2001:

- The data still show an excess, at the level of $2.1 \sigma$, over the expected SM background, mainly due to ALEPH data and to the four-jet final state (to be compared with the $2.9 \sigma$ excess in the preliminary data of November 2000).

- The maximum likelihood occurs at $m_{H}=115.6 \mathrm{GeV}$, with $3.5 \%$ probability of a fluctuation of the SM background.

- The lower bound on the Higgs mass is $m_{H}>114.1 \mathrm{GeV}$ at $95 \%$ c.l., to be compared with an expected bound of $115.4 \mathrm{GeV}$.

- Three out of the four LEP experiments have not yet released their final results at the time of this Conference: the final combination of the LEP results is expected for the end of 2001 .

\subsection{SM fits to the Higgs mass}

Besides direct searches, additional information on the SM Higgs boson come from the fits to $m_{H}$ based on electroweak precision data, whose experimental aspects are discussed in another talk at this Conference 5 . A popular summary of the available information $\mathrm{B}$ is the famous 'blueband' plot of the LEP Electroweak Working Group, displayed in Fig. 1: it gives the $\Delta \chi^{2}=\chi^{2}-\chi_{\min }^{2}$ of the global SM fit to electroweak precision data as a function of $m_{H}$. As evident from Fig. 1, the fit clearly favours a light Higgs. The default fit, represented by the solid curve in Fig. 1, gives $m_{H}=88_{-35}^{+53} \mathrm{GeV}$ and $m_{H}<196 \mathrm{GeV}$ at $95 \%$ c.l.. An alternative fit (see below for an explanation), represented by the dashed

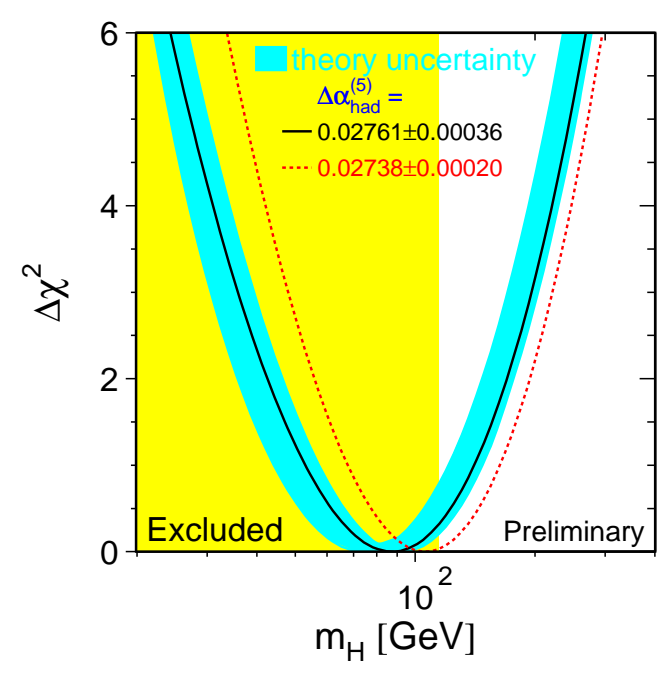

Figure 1. $\Delta \chi^{2}$ as a function of $m_{H}$ from the global fit to the SM. The region excluded by the direct searches at LEP is also shown.

line, gives $m_{H}<222 \mathrm{GeV}$ at $95 \%$ c.l. and a slightly higher central value. The band represents a (debatable) estimate of the theoretical uncertainty. Notice that the fit does not include the information coming from direct searches. Notice also that, in both fits, more than half of the $\chi^{2}$ curve falls in the shaded region, excluded at $95 \%$ c.l. by direct searches.

Given the importance of the issue, it is worth examining in more detail how the preference for a light SM Higgs arises. For given values of the remaining SM input parameters, precise electroweak data combined with updated theoretical calculations give logarithmic sensitivity to $m_{H}$, mostly via two pseudo-observables: the leptonic effective electroweak mixing angle, $\sin ^{2} \theta_{\text {eff }}^{\text {lept }}=$ $\left(1-v_{l} / a_{l}\right) / 4$, and the mass of the $W$ boson, $m_{W}$. There are still small theoretical uncertainties in the evaluation of radiative corrections, in principle reducible by more refined calculations. A recent progress along these lines is the calculation $\mathrm{Z}$ of the com- 
plete fermionic two-loop contribution to $m_{W}$, but other calculations of the same order are still missing. Larger uncertainties come from non-negligible errors in other parameters entering the fit. An important one is the hadronic contribution to the running of the electromagnetic coupling constant, $\Delta \alpha_{\text {had }}^{(5)}$, as extracted from a dispersion integral over a parametrization of the measured crosssection for $e^{+} e^{-} \rightarrow$ hadrons, including the recent data $\mathrm{O}$ from BES and CMD-2. A conservative, 'data-driven' fit 9 gives

$$
\Delta \alpha_{\text {had }}^{(5)}\left(m_{Z}\right)=0.02761 \pm 0.00036,
$$

corresponding to the 'default' input of the blueband plot, whereas a more aggressive, 'theory-driven' fit 10 , corresponding to the 'alternative' input of the blueband plot, gives

$$
\Delta \alpha_{\text {had }}^{(5)}\left(m_{Z}\right)=0.02738 \pm 0.00020 .
$$

There are many other determinations, as reviewed for example in Ref. 11, all consistent with the previous ones, but with a tendency to be closer to Eq. (3) than to Eq. (4). The second important uncertainty in the input parameters is the one associated with the experimental determination of the top quark mass from the CDF and D0 experiments at Fermilab 12 :

$$
m_{\text {top }}=174.3 \pm 5.1 \mathrm{GeV} .
$$

The individual experimental determinations of $\sin ^{2} \theta_{\text {eff }}^{\text {lept }}$ and $m_{W}$, with the corresponding theoretical predictions and uncertainties, as taken from Ref. 6 , are displayed in Figs. 2 and 3 .

A careful inspection of Figs. 13 reveals that the SM fit is not entirely a bed of roses, as stressed, for example, in Ref. 13 (on the basis of the data available in Winter 2001). First, the quality of the overall fit turns out to be acceptable but not exceptional, $\chi^{2} / d o f=22.9 / 15$, corresponding to a 'probability' of $8.6 \%$. The main reason for this can be seen from Fig. 2: there is a systematic tendency of the hadronic asymmetries $(b \bar{b}$ and $c \bar{c}$ forward-backward asymmetry plus

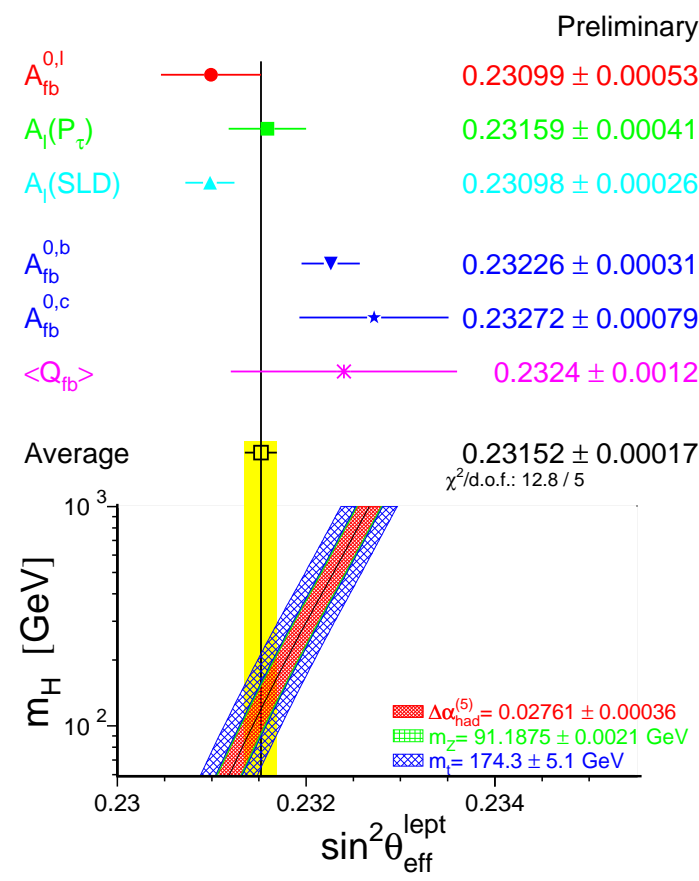

Figure 2. Determination of $\sin ^{2} \theta_{\text {eff }}^{\text {lept from the asym- }}$ metry measurements. The SM prediction is also shown, as a function of $m_{H}$, with the uncertainties from $\Delta \alpha_{h a d}^{(5)}\left(m_{Z}\right)$ and $m_{t}$ added linearly.

quark charge asymmetry) to give larger values of $\sin ^{2} \theta_{\text {eff }}^{\text {lept }}$ (and of $m_{H}$ ) than the leptonic asymmetries (forward-backward asymmetry, $\tau$ polarization asymmetry, SLD left-right asymmetry). The average of the hadronic determinations alone gives $\sin ^{2} \theta_{\text {eff }}^{\text {lept }}($ had $)=$ $0.23230 \pm 0.00029$, the average of the leptonic ones $\sin ^{2} \theta_{\text {eff }}^{\text {lept }}(l e p)=0.23113 \pm 0.00021$, corresponding to a discrepancy at the level of $3.3 \sigma$. This effect was larger in Winter 2001: the change is mostly due to a $-0.5 \sigma$ shift of the $b \bar{b}$ forward-backward asymmetry, $A_{F B}^{0, b}$, after a new DELPHI analysis based on a neural network to tag the b-charge, and an improvement in the jet-charge measurement of the ALEPH analysis. The most precise hadronic determination comes indeed from $A_{F B}^{0, b}$, and has now a pull of $2.9 \sigma$ with respect to the central value of the global SM fit. Keeping in mind the possibility of a statisti- 


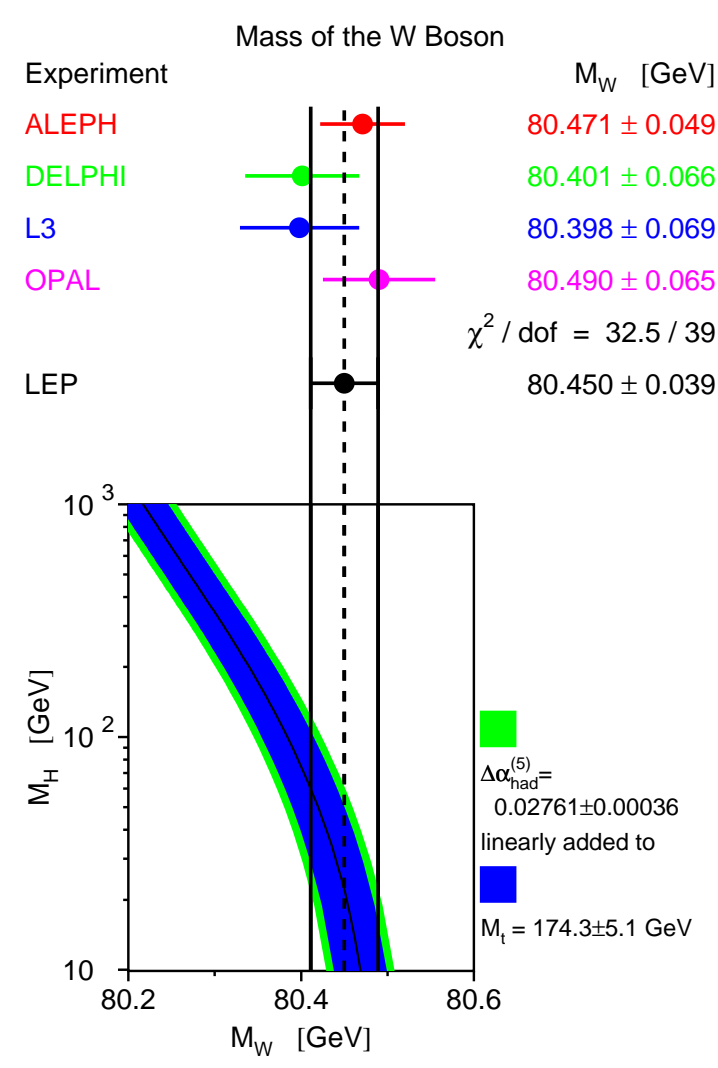

Figure 3. The measurements of $m_{W}$ at LEP. The lower plot shows the SM prediction, as a function of $m_{H}$, with the uncertainties from $\Delta \alpha_{h a d}^{(5)}\left(m_{Z}\right)$ and $m_{t}$ added linearly.

cal fluctuation, this can be viewed as a small problem either for the SM or for the experimental analyses. Radical modifications of the $Z b \bar{b}$ vertex appear unlikely, given the fact that $A_{b}$ from SLD and $R_{b}$ are well-behaved. Also, measuring $A_{F B}^{0, b}$ is a very delicate experimental task, since flavor and charge of the b-quarks need to be tagged simultaneously, with more complicated systematics than in the measurement of $R_{b}$. On the other hand, all the experimental determinations of $m_{W}$ are in good agreement and point to a light Higgs boson: those of Fig. 3 can be combined with the ones from the UA2, CDF and D0 experiments at $p \bar{p}$ colliders, giving $m_{W}=80.454 \pm 0.060$, to produce a global world average $m_{W}=80.451 \pm 0.033$. Notice that, with the present errors, the main parametric uncertainty affecting the theoretical determination of $m_{W}$ is the one coming from $m_{t}$, whereas $m_{t}$ and $\Delta \alpha_{\text {had }}^{(5)}\left(m_{Z}\right)$ give comparable uncertainties in the theoretical determination of $\sin ^{2} \theta_{\text {eff }}^{\text {lept }}$.

Given the small discrepancy between hadronic and leptonic asymmetries, the exercise of looking at what happens, when dropping the hadronic asymmetries from the fit, may not be entirely academical. The result is the following: the quality of the SM fit improves, but the central value of $m_{H}$ is pushed down, so that the consistency of the SM fit to $m_{H}$ with the limits from direct searches becomes marginal [with a significant residual dependence, which should not be forgotten, on $\Delta \alpha_{h a d}^{(5)}\left(m_{Z}\right)$ and $\left.m_{t}\right]$. Is there a SM crisis lurking around the corner? A prudent attitude before answering this question may be appropriate, taking into account that the final heavy-flavor analyses from LEP and improved determinations of $m_{W}$ and $m_{t}$ from the new Tevatron run will be available soon. It may well be, however, that we must wait until the discovery (or the exclusion) of a light Higgs boson to definitively settle the issue.

\subsection{The SM as an effective theory}

If we believed that the SM is the whole story, the talk could end here. However, we all know that the SM cannot be the ultimate theory of elementary particles, valid at arbitrarily high energy scales, since it does not contain a quantum theory of gravitational interactions and some of its couplings are not asymptotically free. Thus, the SM must be seen as an effective field theory, valid up to some physical cut-off scale $\Lambda$, where new physics must be introduced into the theory. On general grounds, $\Lambda$ could be anywhere between the $\mathrm{TeV}$ scale and the Planck scale, $M_{P} \equiv G_{N}^{-1 / 2} / \sqrt{8 \pi} \simeq 2.4 \times 10^{18} \mathrm{GeV}$, where 
$G_{N}$ is Newton's constant, characterizing the observed gravitational interactions. Assuming that the SM correctly identifies the degrees of freedom at the weak scale (this may not be true, as will be discussed later, in the case of the Higgs field), we can write down the most general local Lagrangian compatible with the SM symmetries, classifying the possible operators according to their physical dimension, and scaling all dimensionful couplings by appropriate powers of $\Lambda$. The resulting dimensionless coefficients are then to be interpreted as parameters, which can be either fitted to experimental data or (if we are able to do so) theoretically determined from the fundamental theory replacing the SM at the scale $\Lambda$. Very schematically (and omitting all coefficients and indices, as well as many theoretical subtleties):

$$
\begin{aligned}
\mathcal{L}_{\text {eff }}^{S M}= & {\left[\Lambda^{4}+\Lambda^{2} \Phi^{2}\right]+\left[(D \Phi)^{2}+\bar{\Psi} \not D \Psi\right.} \\
& \left.+F^{\mu \nu} F_{\mu \nu}+F^{\mu \nu} \tilde{F}_{\mu \nu}+\bar{\Psi} \Psi \Phi+\Phi^{4}\right] \\
& +\left[\frac{\bar{\Psi} \Psi \Phi^{2}}{\Lambda}+\frac{\bar{\Psi} \sigma^{\mu \nu} \Psi F_{\mu \nu}}{\Lambda}+\frac{\bar{\Psi} \Psi \bar{\Psi} \Psi}{\Lambda^{2}}\right. \\
& \left.+\frac{\Phi^{2} F^{\mu \nu} F_{\mu \nu}}{\Lambda^{2}}+\ldots\right]
\end{aligned}
$$

where $\Psi$ stands for the generic quark or lepton field, $\Phi$ for the SM Higgs field, $F$ for the field strength of the SM gauge fields, and $D$ for the gauge-covariant derivative. The first bracket in Eq. (6) contains two terms, a cosmological constant term and a Higgs mass term, that are proportional to positive powers of $\Lambda$, and are at the origin of two infamous hierarchy problems. The second bracket in Eq. (6) contains operators with no powerlike dependence on $\Lambda$, but only a milder, logarithmic dependence, due to infrared renormalization effects between the cut-off scale $\Lambda$ and the weak scale. The last bracket in Eq. (6) is the starting point of an expansion in inverse powers of $\Lambda$, and contains operators associated with rare processes, precision tests, neutrino masses, proton decay,

In this framework, an old question 14 can be addressed in the light of present experimental data: given our knowledge of the top quark mass and of the bounds on the Higgs mass, can we put some firm bounds on the cut-off scale $\Lambda$ ? The qualitative aspects of the answer can be appreciated by remembering that, in the SM, the top and Higgs masses are associated with the largest Yukawa coupling $h_{t}$ and with the quartic Higgs selfcoupling $\lambda$, respectively, via tree-level relations of the form $m_{t} \propto h_{t} v$ and $m_{H}^{2} \propto \lambda v^{2}$, where $v$ is the vacuum expectation value of the Higgs field. Also, the scale-dependence of $\lambda$ is controlled by the renormalization group equation

$$
\frac{d \lambda}{d \log Q}=\frac{3}{16 \pi^{2}}\left(\lambda^{2}+\lambda h_{t}^{2}-h_{t}^{4}\right)+\ldots,
$$

where $Q$ is the renormalization scale and the dots stand for smaller one-loop contributions, controlled by the electroweak gauge couplings, and higher-order contributions. For any given values of $m_{t}$ and $\Lambda$, we can extract a 'triviality' upper bound on $m_{H}$ observing that, if $m_{H}$ is too large, $\lambda(Q)$ blows up at a scale $Q_{0}<\Lambda$, developing a Landau pole. This leads to some well-known constraints, supported by more rigorous arguments and by lattice calculations: $m_{H}<$ $200 \mathrm{GeV}$ if $\Lambda \sim M_{P}, m_{H}<600 \mathrm{GeV}$ if $\Lambda \sim 1 \mathrm{TeV}$. Similarly, we can extract a 'stability' lower bound on $m_{H}$ by observing that, if $m_{H}$ is too small, then $\lambda(Q)$ becomes negative at $Q_{0}<\Lambda$, and another minimum of the SM potential develops at $\langle\phi\rangle \sim Q_{0}$.

Since the results of the previous subsection point to rather small values of $m_{H}$, the presently hot issue is the stability bound, recently revisited in 15 . When implementing the stability bound, three options are possible: 1) we can require absolute stability, i.e. the correct electroweak vacuum must have lower energy than the 'wrong' vacuum; 2 ) we can require stability with respect to high-temperature fluctuations in the cosmo- 
logical evolution of the early Universe; 3 ) we can require stability with respect to quantum fluctuations at approximately zero temperature. The latter is the most conservative option, and amounts to requiring that the lifetime of the correct electroweak vacuum should be larger than the present age of the Universe, $T_{U} \sim 10^{10}$ yrs. The present results are illustrated 10 in Fig. A, where

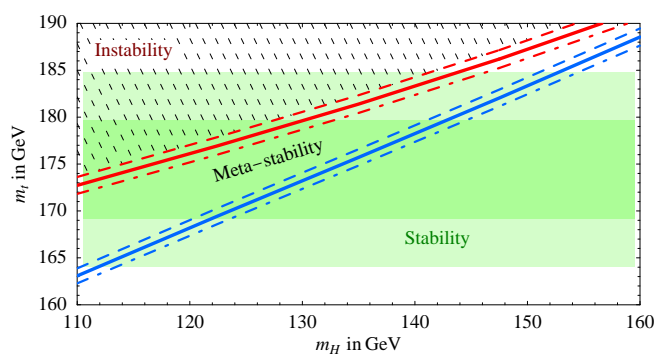

Figure 4. Instability, meta-stability and stability regions of the SM vacuum in the $\left(m_{H}, m_{t}\right)$ plane, for $\alpha_{S}\left(m_{Z}\right)=0.118$ (solid curves) \pm 0.002 (dashed and dash-dotted curves). The shaded area indicates the experimental range fro $m_{t}$, Eq. (5), at $1 \sigma$ (darker) and $2 \sigma$ (lighter)

$\alpha_{S}\left(m_{Z}\right)=0.118 \pm 0.002$ and $\Lambda=M_{P}$ have

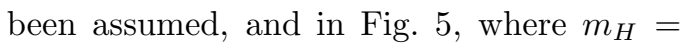

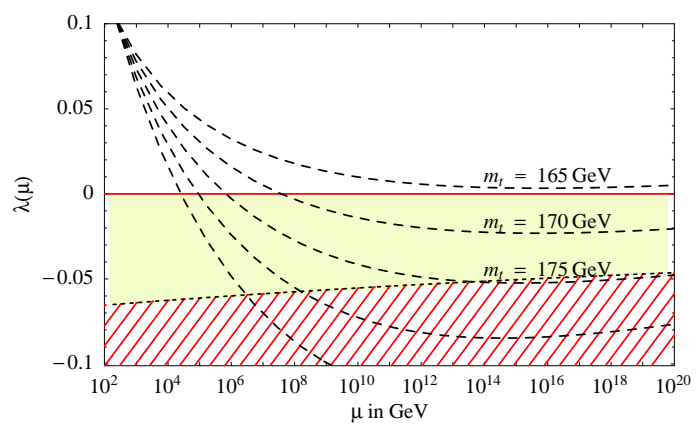

Figure 5. Running of the quartic Higgs coupling $\lambda(\mu)$ for $m_{H}=115 \mathrm{GeV}, m_{t}=165,170,175,180,185 \mathrm{GeV}$ and $\alpha_{S}\left(m_{Z}\right)=0.118$. Absolute stability $\left[\lambda\left(M_{\text {weak }}\right)>0\right]$ is still possible if $m_{t}<166 \mathrm{GeV}$. The hatched region is excluded by the meta-stability bound.

$115 \mathrm{GeV}$ and $\alpha_{S}\left(m_{Z}\right)=0.118$ have been assumed. If we set $\Lambda \sim M_{P}$ (its maximum sensible value) and $m_{H}=115 \mathrm{GeV}$ (close to its minimum allowed value and to the location of the slight experimental effect discussed in subsection 1.1), then option (1) leads to $m_{t}<(166 \pm 2) \mathrm{GeV}$. After a new complete one-loop calculation of the tunneling probability at zero temperature, option (3) leads to $m_{t}<(175 \pm 2) \mathrm{GeV}$, still in full agreement with the data. Therefore, it may be premature to claim evidence of new physics below $M_{P}$ from SM vacuum stability, even if we are at the border of the allowed region, a situation for which possible theoretical reasons have been suggested 16 .

\section{MSSM (The Dogma?)}

In the SM effective Lagrangian of Eq. 6, the mass term for the Higgs field has a quadratic dependence on the cut-off scale $\Lambda$. When we try to extrapolate the SM to scales much higher than the weak scale, this gives rise to the infamous gauge hierarchy problem. The natural solution to this problem is to introduce new physics close to the weak scale. The present best candidate for such new physics is the Minimal Supersymmetric extension of the Standard Model (MSSM), extensively discussed in another talk at this Conference 17 .

\subsection{Some virtues of the MSSM}

The main virtue of the MSSM is that, if the mass splittings $\Delta m_{\text {susy }}$ that break supersymmetry (SUSY) are of the order of the weak scale $M_{\text {weak }} \sim 1 \mathrm{TeV}$, then its cut-off scale can be naturally taken to be $\Lambda_{M S S M}=$ $\Lambda_{\text {susy }}^{2} / \Delta m_{\text {susy }}$, where $\Lambda_{\text {susy }}$ is the scale of spontaneous SUSY breaking. In hiddensector supergravity models, where $\Lambda_{\text {susy }} \sim$ $\sqrt{M_{\text {weak }} M_{P}}$, such cut-off scale can then be pushed very close to $M_{P}$ (this is not true if SUSY breaking occurs at lower scales, as in 'gauge-mediated' models).

Another virtue of the MSSM is that, in contrast with other possible solutions of the hierarchy problem, it is generically as good as 
the SM in complying with electroweak precision data. This is due to the fact that the soft SUSY-breaking mass terms do not break the $S U(2)_{L} \times U(1)_{Y}$ gauge symmetry. Indeed, it was recently observed 18 that, if sneutrinos and charged sleptons (and, to a lesser extent, charginos and neutralinos) have masses close to their present experimental bounds, then the MSSM may lead to an improved consistency between direct and indirect bounds on the Higgs mass, when the hadronic asymmetries are left out of the global fit. This result is illustrated in Figs. 6 and 7 , drawn in planes

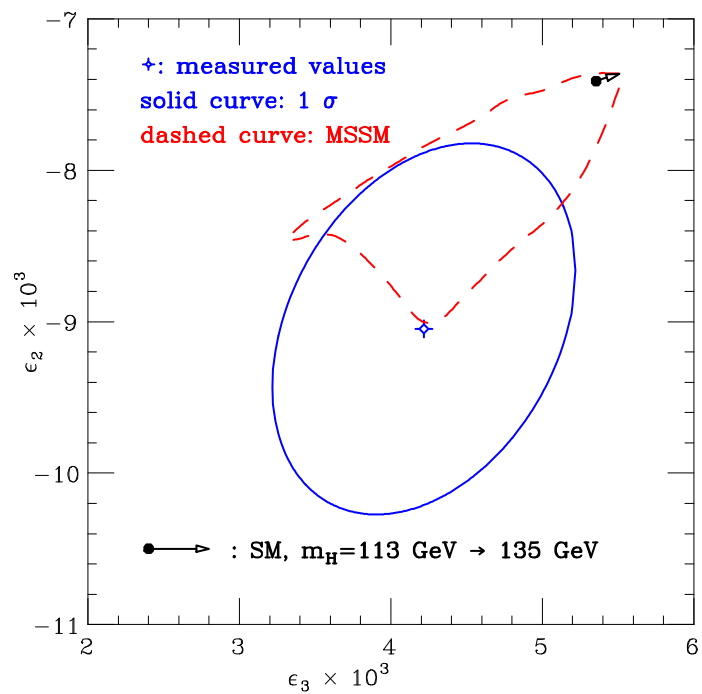

Figure 6. Measured values (cross) of $\epsilon_{3}$ and $\epsilon_{2}$, with their $1 \sigma$ region (solid ellipse), obtained from $m_{W}, \Gamma_{l}$, $\sin ^{2} \theta_{\text {eff }}^{\text {lept }}(l e p)$ and $R_{b}$. The area inside the irregular curve represents the MSSM prediction for $m_{\tilde{e}_{L}}$ between 96 and $300 \mathrm{GeV}, m_{\chi^{ \pm}}$between 105 and $300 \mathrm{GeV},|\mu|<1 \mathrm{TeV}, \tan \beta=10, m_{\tilde{e}_{R}}=1 \mathrm{TeV}$ and $m_{A}=1 \mathrm{TeV}$.

characterized by two of the three flavourindependent parameters $\left(\epsilon_{1}, \epsilon_{2}, \epsilon_{3}\right)$ that are often used in non-SM fits to precision data. We remind the reader that $\epsilon_{1}$, related to Veltman's parameter $\delta \rho$, is mainly controlled by $m_{t}, \epsilon_{2}$ is particularly sensitive to $m_{W}$ and $\epsilon_{3}$ is mainly controlled by $\sin ^{2} \theta_{\text {eff }}^{\text {lept }}(l e p)$. Only the $W$ mass, the leptonic $Z$ width, the leptonic asymmetries and the ratio $R_{b}$ have been

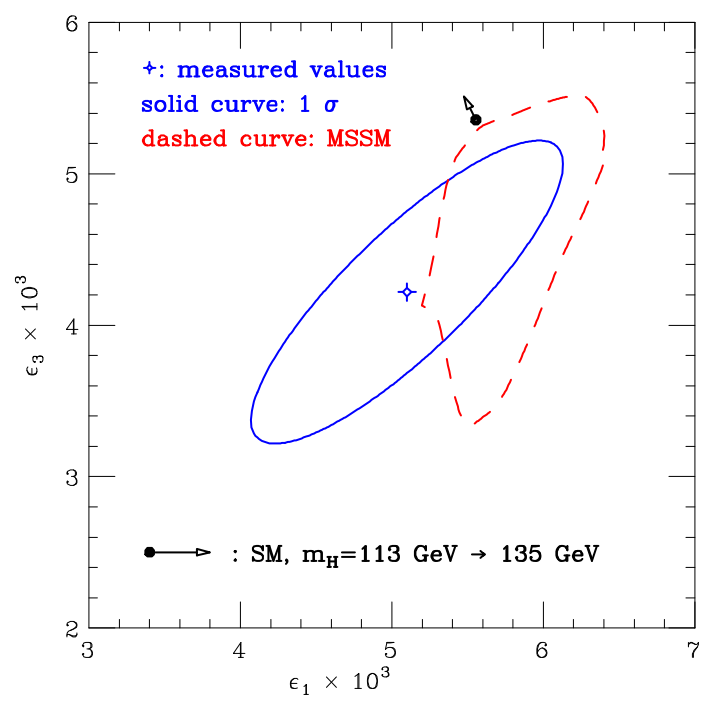

Figure 7. The same as in Fig. B but for $\epsilon_{1}$ and $\epsilon_{3}$.

included in the fit. The elliptic solid contours represent the region allowed by the data at the $1 \sigma$ level. The irregular dashed contours enclose the typical MSSM predictions for a light spectrum. The fat dot with an arrow shows the SM prediction for a Higgs mass varying between $113 \mathrm{GeV}$ and $135 \mathrm{GeV}$. We can see that, for a light MSSM spectrum, the agreement between data and theoretical predictions can improve.

Another important piece of indirect evidence in favour of the MSSM is the fact that, when combined with a condition on the grand unification of all gauge couplings and with the hypothesis of a 'desert' between the weak scale and the grand unification scale, it leads 19 to one successful prediction for the gauge couplings at the weak scale. To gauge the significance of this success, we can perform a simple-minded but illuminating exercise. We can consider the one-loop renormalization group equation for the running gauge coupling constants

$$
\frac{d \alpha_{A}}{d \log Q}=\frac{b_{A}}{2 \pi} \alpha_{A}^{2}+\ldots, \quad(A=1,2,3),
$$

where $b_{A}$ are the one-loop beta-function co- 
efficients, determined by the gauge quantum numbers of the particle spectrum at the weak scale. If we are agnostic about the precise value of the unified gauge coupling and of the grand unification scale, but we assume the normalization of the $U(1)_{Y}$ gauge coupling suggested by the simplest grand-unified models, we can perform a unification test by considering the only variable controlling the prediction for the gauge couplings at the weak scale, the ratio $B \equiv\left(b_{3}-b_{2}\right) /\left(b_{2}-b_{1}\right)$. The SM value of this ratio is $B_{S M} \simeq 0.53$, its experimental value is $B_{\text {exp }} \simeq 0.71$, and the MSSM value is $B_{M S S M} \simeq 0.72$. A reasonable error estimate is $\Delta B \sim 0.03$, completely dominated by the the fact that we do not know the details of the MSSM spectrum and of the spectrum of the underlying theory around the grand unification scale. This is an impressive success, and it is difficult to believe that it is accidental and that we are being fooled by a malicious Nature and by theorists. Any other extension of the SM claiming to be better than the MSSM must face this important phenomenological hint.

\subsection{The MSSM Higgs sector}

If we take seriously the MSSM, then it is important to extract its predictions for the Higgs sector. As is well known, the MSSM Higgs sector contains two complex doublets, which after gauge symmetry breaking give rise to five physical degrees of freedom, three neutral $(h, H, A)$ and two charged $\left(H^{ \pm}\right)$. The prediction of SUSY is that the MSSM Higgs sector depends, at the tree level, only on known SM parameters and two more parameters, for example $m_{A}$ and $\tan \beta \equiv v_{2} / v_{1}$. After including quantum corrections, the predictions of SUSY are not lost, but the dependences become more complicated and involve all the rest of the MSSM spectrum, in particular the parameters of the top-stop sector 20. An intense theoretical effort has been devoted over the last years to the precise computa- tion of the MSSM Higgs properties, and we are now at the stage where the calculation of the most important two-loop corrections is being completed. When the top quark mass will be known more precisely, these calculations will be important for reliably comparing models of SUSY breaking with the available bounds on the spectrum. Of course, the relevance of all this could increase further if and when SUSY particles and SUSY Higgs bosons will be found. So far, two-loop corrections to the neutral Higgs boson masses have been computed mostly in the limit of vanishing momentum on the external lines of the Higgs and gauge boson propagators. In this limit, analytical formulae at $\mathcal{O}\left(\alpha_{t} \alpha_{S}\right)$ are available, for arbitrary values of the relevant MSSM parameters 21, and have been implemented in computer codes. As for the $\mathcal{O}\left(\alpha_{t}^{2}\right)$ corrections, which can be of comparable numerical importance, at the time of this Conference there are only partially analytic formulae 22 for $m_{h}$, valid in the limit $m_{A} \gg m_{Z}$. The general calculation of the $\mathcal{O}\left(\alpha_{t}^{2}\right)$ corrections (in the zero-momentum limit) has been recently completed 23 and agrees with Ref. 22 in the appropriate limit. The effects of the $\mathcal{O}\left(\alpha_{t}^{2}\right)$ corrections is illustrated in Fig. 8 , taken from Ref. 22. We can see that these corrections can be sizeable, increasing $m_{h}$ by several $\mathrm{GeV}$ in the case of large mixing in the stop mass matrix.

Armed with the relevant radiative corrections [the $\mathcal{O}\left(\alpha_{t}^{2}\right)$ ones have not yet been adequately implemented in the codes, but will presumably be included in the final LEP analyses], experimentalists have searched for direct signals of the MSSM Higgs bosons, as reviewed in another talk at this Conference 3 and described in more detail in Ref. 24. The small Higgs signal in the SM analysis has its counterpart in the MSSM analysis: some excesses at the $\sim 2 \sigma$ level are reported both in the $e^{+} e^{-} \rightarrow h A$ channel, at $\left(m_{h}, m_{A}\right) \sim(83,83),(93,93) \mathrm{GeV}$, and in the $e^{+} e^{-} \rightarrow h Z$ channel, for $m_{h} \sim 97,115 \mathrm{GeV}$. 

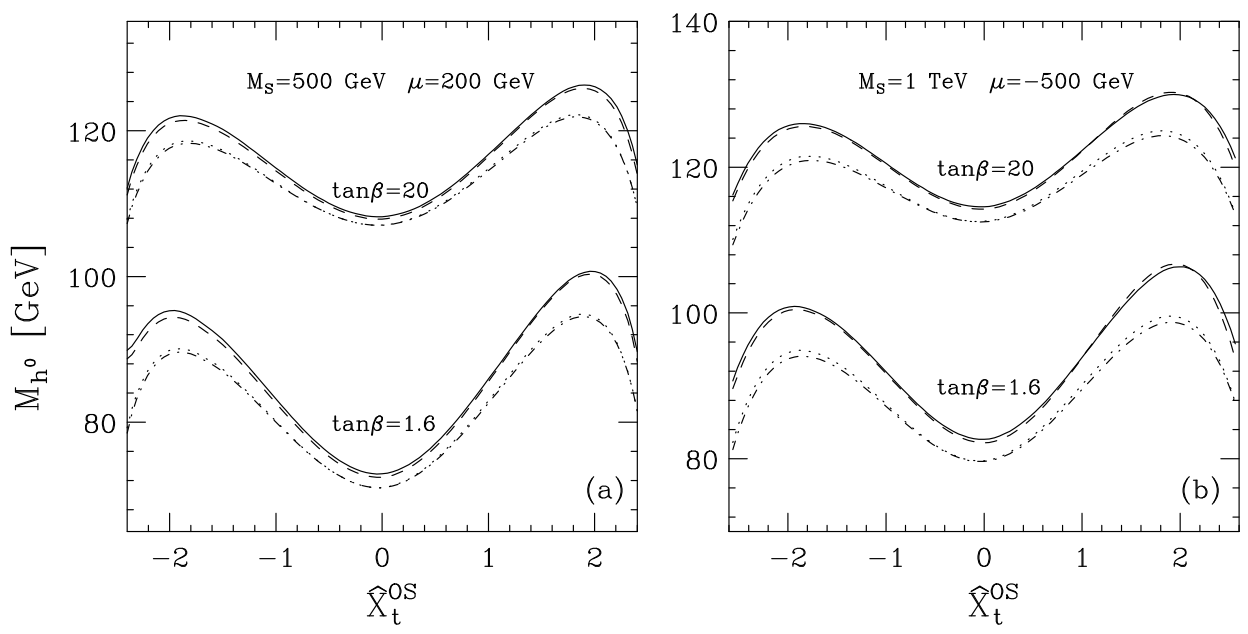

Figure 8. The mass $m_{h}$ vs. the stop mixing parameter $\hat{X}_{t}^{O S}$, for some representative values of the remaining MSSM parameters. The two-loop corrections are included either at $\mathcal{O}\left(\alpha_{t} \alpha_{S}\right)$ (lower lines) or at $\mathcal{O}\left(\alpha_{t} \alpha_{S}+\alpha_{t}^{2}\right)$ (upper lines). The fine structure corresponds to two different methods of implementing the corrections.

The lower bounds on the MSSM Higgs masses are notoriously difficult to illustrate, due to their dependence on many parameters. Examples of exclusion plots are presented in Figs. 9 and 10, for a representative choice of MSSM parameters. In a 'benchmark' case characterized by a large mixing in the stop mass matrix, which should lead to conservative bounds on $\left(m_{h}, m_{A}\right)$ and on $\tan \beta$, the data have been interpreted 24 in terms of the following exclusion regions at $95 \%$ c.l.: $\left(m_{h}, m_{A}\right)<(91.0,91.9) \mathrm{GeV}$ and $\tan \beta<$ 2.4. For small stop mixing, the limits are typically stronger.

There are other recent interesting studies of the MSSM Higgs sector that would deserve to be discussed. There is just the time to briefly mention them, referring the reader to the corresponding papers.

There is a new experimental analysis of the Tevatron data 25, on the search for $p \bar{p} \rightarrow b \bar{b} \varphi \rightarrow b \bar{b} b \bar{b}(\varphi=h, H, A)$. It leads to non-trivial constraints on the Higgs spectrum, in the region of the MSSM parameter space where the bottom Yukawa coupling is strongly enhanced with respect to its SM value, $\tan \beta>40-50$.
Some recent theoretical studies 26 have considered the possibility of radiatively induced $\mathrm{CP}$-violating effects in the Higgs sector, coming from explicit $\mathrm{CP}$-violating phases in the squark-gluino sector, and have analyzed the resulting complications in the discussion of the MSSM Higgs searches. Other theoretical studies 27 have examined the implications of the experimental bounds on the MSSM Higgses for different models of SUSY-breaking 'mediation'.

\subsection{Some weak points of the MSSM}

It would be misleading to end this section without mentioning that, besides its virtues, the MSSM has also, in our present view, a number of weak points.

To begin with, the MSSM with its soft SUSY breaking provides only an incomplete, technical solution of the hierarchy problem, since the overall mass scale of the soft terms is set 'by hand'. These soft terms also introduce a very large number of free parameters into the model: this problem is going to stay with us until when a standard model for spontaneous supersymmetry breaking will 


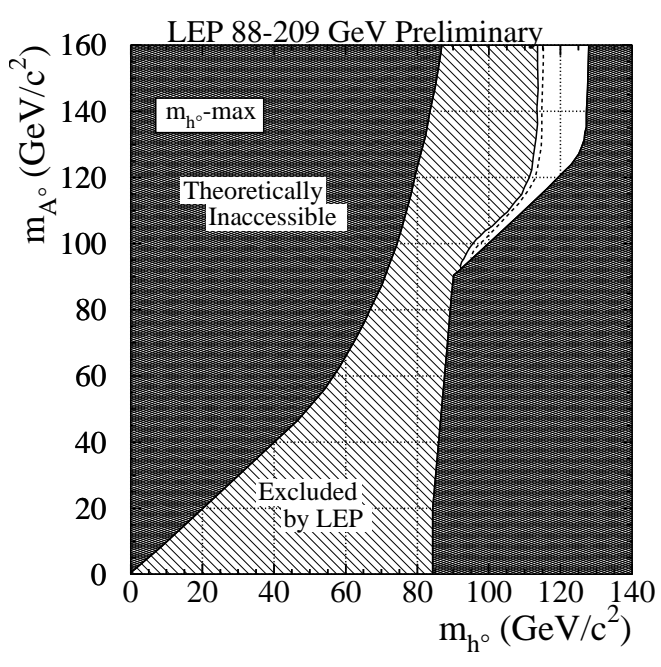

Figure 9. The MSSM exclusion region in the $\left(m_{h}, m_{A}\right)$ plane, for the ' $m_{h}-\max$ ' benchmark scenario. The central region is excluded by LEP searches, the lateral ones are theoretically inaccessible in such a scenario.

emerge and/or SUSY particles will be found.

More seriously, after many years of experimental searches at increasing energy scales, which explored a large part of the theoretically most appealing region from the point of view of the hierarchy problem, no direct experimental hint for the existence of the MSSM Higgs or SUSY particles has been found.

Taking all this into account, we should not take the MSSM as a dogma for the new physics at the weak scale, but keep an open mind for the possible alternatives.

\section{Can we do without a light Higgs? (The Heresy?)}

This part of the talk will touch an issue that often triggers heated discussions: can we do without a light Higgs? Some people view this as a heresy, some others almost take it for granted, so it is worth reviewing it, even if the state of affairs has not changed in an impor-

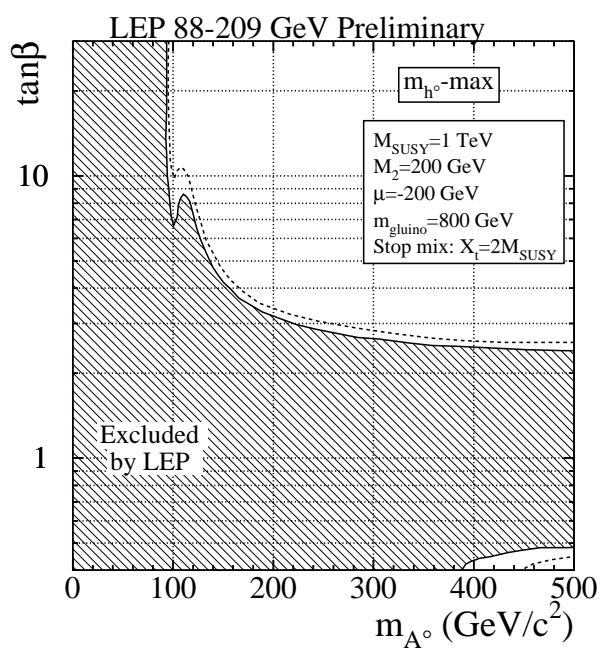

Figure 10. The MSSM region of the $\left(m_{A}, \tan \beta\right)$ plane excluded by LEP searches, for the ' $m_{h}-\max$ ' benchmark scenario.

tant way during the last year. Since, to the taste of most theorists, there is no satisfactory model without a light elementary Higgs, we may take an agnostic point of view and work at the level of an effective field theory.

The most drastic departure from the SM consists in getting rid of the elementary Higgs field, and having the electroweak gauge symmetry $S U(2)_{L} \times U(1)_{Y}$ non-linearly realized (so doing, of course, we define a nonrenormalizable effective theory whose cut-off scale cannot be much above the $\mathrm{TeV}$ scale). This approach has a long history, from the pioneering papers of Ref. 28 to some recent phenomenological discussions 29 after the LEP and Tevatron data. In this approach, the effective Lagrangian is constructed from the Goldstone bosons $w^{a}$ associated with electroweak symmetry breaking, assembled into the group element $\Sigma=\exp \left(2 i w^{a} \tau^{a} / v\right)$, where $v \simeq 256 \mathrm{GeV}$. Concentrating on the terms that can affect the $W$ and $Z$ propagators, thus playing a major role in the discussion 
of electroweak precision tests, we can write

$$
\begin{aligned}
\mathcal{L}_{\text {eff }} & =\frac{v^{2}}{4} \operatorname{Tr}\left(D_{\mu} \Sigma D^{\mu} \Sigma^{\dagger}\right) \\
& +\sum_{i} \tilde{c}_{i} \widetilde{\mathcal{O}}_{i}(\Sigma, \widetilde{\Lambda}, \ldots)
\end{aligned}
$$

where

$$
D_{\mu} \Sigma=\partial_{\mu} \Sigma+i g W_{\mu}^{a} \tau^{a} \Sigma-i g^{\prime} \Sigma B_{\mu}^{3} \tau^{3}
$$

is the covariant derivative. The first term in (9) describes the $W$ and $Z$ masses, as can be seen immediately in the unitary gauge $\Sigma=1$. The higher-order operators $\widetilde{\mathcal{O}}_{i}$ are scaled by appropriate powers of the cut-off $\widetilde{\Lambda}$ of this Higgsless theory, and are characterized by dimensionless coefficients $\tilde{c}_{i}$.

A less drastic approach consists in keeping the elementary Higgs field $\phi$, so that $S U(2)_{L} \times U(1)_{Y}$ can be linearly realized, but in allowing the most general set of nonrenormalizable operators compatible with the electroweak gauge symmetry and with Poincaré invariance. Also this approach has a long history, from the early paper of Ref. 30 to other recent phenomenological discussions 31 after the LEP and Tevatron data. In this case, the appropriate effective Lagrangian is

$$
\mathcal{L}_{\text {eff }}=\mathcal{L}_{S M}(\phi)+\sum_{i} c_{i} \mathcal{O}_{i}(\phi, \Lambda, \ldots),
$$

where $\Lambda$ is the cut-off and $c_{i}$ are the dimensionless coefficients of the various operators $\mathcal{O}_{i}$.

In both approaches, the theoretical expressions for the two key pseudo-observables in the fits, $\sin ^{2} \theta_{\text {eff }}^{\text {lept }}$ and $m_{W}$, differ from the $\mathrm{SM}$ ones. For given values of all the other parameters, in the SM they are just functions of $m_{H}$, with their leading dependences proportional to $\log \left(m_{H} / m_{Z}\right)$. In these new frameworks, the dependences become more complicated:

$$
\log \frac{m_{H}}{m_{Z}} \rightarrow\left\{\begin{array}{l}
\log \frac{\widetilde{\Lambda}}{m_{Z}} \\
\log \frac{m_{H}}{m_{Z}}
\end{array}+K_{\theta, W}\left(\begin{array}{c}
\tilde{c}_{i}, \tilde{\Lambda} \\
c_{i}, \Lambda
\end{array}\right) .\right.
$$

In the Higgsless effective theory, the logarithmic dependence on $\widetilde{\Lambda} / m_{Z}$, generated by renormalization, must be combined with finite contributions $K_{\theta}$ (for $\sin ^{2} \theta_{\text {eff }}^{\text {lept }}$ ) and $K_{W}$ (for $m_{W}$ ). A similar phenomenon occurs in the effective theory with the Higgs field, with the only difference that the logarithmic dependence is on $m_{H} / m_{Z}$. At the level of both effective theories, $K_{\theta, W}$ depend on the cutoff and on the unknown dimensionless coefficients of the higher-dimensional operators, on which we can get reliable information only if we know about the underlying fundamental theory. With the present data, it is still possible to have $\widetilde{\Lambda}\left(m_{H}\right) \gg m_{Z}$ without excessive fine-tuning of the quantities $K_{\theta, W}$.

A more careful analysis, however, reveals the present advantage of the light Higgs hypothesis. First, it must be said that, despite a lot of effort, so far there are no good candidates for the underlying theory that realizes the desired situation, i.e. the phenomenologically correct magnitudes and signs of $K_{\theta}$ and $K_{W}$, without disrupting the predictions for other observables, and avoiding 'ad hoc' theoretical constructions. Also, it can be immediately seen, in the linear realization, that there is an obvious correlation: if we increase $m_{H}$ we must correspondingly decrease $\Lambda$, and tune the coefficients $c_{i}$, to keep agreement with the data: then $m_{H} \sim m_{Z}$ and $\Lambda \gg m_{Z}$ looks as the most natural solution! There was a recent survey 32 of models that may evade the constraint of having a light Higgs with $m_{H} \sim m_{Z}$. Three classes of models were identified, making reference to the $(S, T)$ parameters, analogous to the $\left(\epsilon_{3}, \epsilon_{1}\right)$

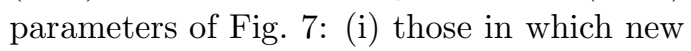
physics can produce negative contributions to the $S$ parameter; (ii) those with a new vector bosons $Z^{\prime}$ close to the weak scale; (iii) those in which new physics can produce positive contributions to the $T$ parameter. Without going to the details, the important point is that all these models exhibit a rich phenomenology around the TeV scale, accessible to accelerators such as the Tevatron 33 , the LHC 34 and a possible high-energy lin- 
ear $e^{+} e^{-}$collider 35 .

\section{New theoretical ideas (Crackpot religions?)}

Electroweak symmetry breaking is a testing ground for new ideas that are restlessly explored by adventurous theorists, despite the fact that some of their most conservative colleagues may view them (in the words of Monty Python) as 'crackpot religions'. It is appropriate to comment here on some ideas that have been very actively explored in the last years, focusing on the aspects that are most strictly related with Higgs physics.

The two big hierarchy problems of our present theories are the cosmological constant problem and the gauge hierarchy problem, related with the two operators of dimension $d<4$ in the SM effective Lagrangian, conveniently rewritten as:

$$
\mathcal{L}_{\text {eff }}^{S M}=\Lambda_{\text {cosm }}^{4}+\Lambda_{\text {weak }}^{2} \phi^{2}+\ldots .
$$

We must explain why the scales of the vacuum energy and of the Higgs mass satisfy the phenomenological bounds $\Lambda_{\text {cosm }} \sim$ $\mathcal{O}\left(10^{-3} \mathrm{eV}\right)$ (as discussed in another talk at this Conference 36$)$ and $\Lambda_{\text {weak }} \sim \mathcal{O}(1 \mathrm{TeV})$, with the intriguing numerical coincidence $\Lambda_{\text {cosm }} \sim \Lambda_{\text {weak }}^{2} / M_{P}$.

Are the two hierarchy problems related? It turns out that they are in supergravity and superstring theories, where supersymmetry becomes a local symmetry and gravity is automatically included. In these theories, formulating an acceptable model for SUSY breaking is difficult, precisely because the problem of the weak scale (most probably linked to the scale of SUSY-breaking masses) and the problem of the cosmological constant scale must be addressed at once.

Many theorists feel that models formulated in more than four space-time dimensions may offer unconventional solutions to these problems and, perhaps, some exotic phenomenology to be explored experimen- tally. Since these models are the subject of another talk at this Conference 37 , some comments related with electroweak symmetry breaking and the gauge hierarchy problem will be sufficient here (for other recent reviews and references on extra dimensions, see e.g. Ref. 38 ).

One of the most interesting features of models with extra dimensions is the fact that the hierarchy $M_{\text {weak }} / M_{P}$ can be linked with some geometrical object, in the simplest case a compactification radius $R$ characterizing the size of one or more compactified dimensions. Such a relation is strongly model-dependent. In toroidal compactifications, we can get power-like relations such as $\left(M_{\text {weak }}^{2} / M_{P}^{2}\right) \propto R^{-n}$, where $n$ is an integer and the dimensionful proportionality coefficient is model-dependent. In 'warped' compactifications, we can get exponential relations of the form $\left(M_{\text {weak }} / M_{P}\right) \sim$ $\exp \left(-M_{P} R\right)$. The gauge hierarchy problem is then reformulated in a very interesting way: it amounts to understanding the stability and the dynamical origin of the value of the radius $R$ that fits the phenomenological value of $M_{\text {weak }}$. There is no compelling idea so far in this direction, but some intriguing features are emerging and are at the center of an intense theoretical activity.

Before describing some of the possibilities, it is worth mentioning that the problem of determining $R$ is analogous to the problem of understanding the stability and the dynamical origin of $\Delta m_{\text {susy }}$, the scale of SUSY-breaking mass splittings, in conventional, four-dimensional models of spontaneous SUSY breaking. The analogy becomes evident in those (higher-dimensional) superstring 3940 and supergravity 11 models where the radius $R$ does indeed control $\Delta m_{\text {susy }}$.

If there are symmetries of the higherdimensional theory whose breaking is nonlocal in the extra dimensions, symmetrybreaking quantities may be shielded from UV effects, and determined by the infrared dy- 
namics. As an example, the field-dependent one-loop effective potentiał of some superstring 40 (and field-theory 23 ) compactifications does not contain positive powers of the string scale (cutoff scale $\Lambda$ )

$$
V_{1}(R, \phi)=R^{-4}+R^{-2} \phi^{2}+\phi^{4}+\ldots,
$$

where all coefficients have been omitted and the dots stand for logarithmic corrections associated with the infrared running of the couplings. Starting from a higher-dimensional theory whose symmetries forbid a Higgs mass term (and ignoring the radius dynamics), $m_{H}$ and $v=\langle\phi\rangle$ are then calculable in terms of $R$, which may lead to one prediction. If there is no ultraviolet sensitivity, the program may be carried out at the field-theory level 43 , even if higher-dimensional field theories are in general badly non-renormalizable. However, if we lose the guidance of a consistent underlying superstring construction, there are many non-trivial consistency constraints to be satisfied, such as the absence of localized destabilizing divergences and anomalies, whose impact is being actively studied at the time of this Conference 44 .

We could be even more ambitious, and try to determine dynamically also $R$, if we could match the coefficient of the $R^{-4}$ term from the gravitational sector of the higherdimensional theory. If all mass scales of the effective four-dimensional theory are controlled by the radius $R$, which in many compactifications is a classical flat direction because of a spontaneously broken scale invariance, then $R$ could be fixed at a finite nonzero value by a dimensional transmutation mechanism: an infrared fixed point for the $R$-dependent vacuum energy could arise from the interplay between the top-Yukawa and the gauge couplings 45 .

In the context of warped compactifications 37 , an interesting mechanism for stabilizing $R$ at its desired value was suggested in Ref. 46. Since it involves the introduction of an explicit mass parameter, in a theory that had initially a classical scale invariance, it may be regarded as the analogous of soft breaking in the MSSM. This mechanism may be stable and related to a dimensional transmutation via the 'holographic' picture 4 .

Coming back to the main subject of the present talk, what features may emerge for Higgs phenomenology? It may be too early to tell. One possibility is the mixing between the Higgs boson(s), charged under the electroweak gauge symmetry, and the spin-0 fields, neutral under the electroweak gauge symmetry, that are associated with the compactification radius ('radion') or, via supersymmetry, with the Goldstone fermion of spontaneously broken supersymmetry ('sgoldstinos'). Such a mixing may lead to possible enhanced couplings of the Higgs to photons, gluons and invisible particles from the gravitational sector 48 . This is a sufficiently good reason to keep an eye on non-standard Higgs searches 24 that do not assume the SM or MSSM production crosssections and branching ratios.

In summary, the exploration of models with extra dimensions looks as a promising approach, in rapid development, with several controversial issues that have not been fully settled yet, and possible impact both on the theory and on the phenomenology of electroweak symmetry breaking.

\section{The ultimate answer (The Universal Judgement)}

In the course of this talk we have been moving towards more and more speculative scenarios. How can we tell what is the correct one? Fortunately, there is an impressive ongoing experimental program that will be able to tell us the answer.

As discussed in another talk at this Conference 33 , the Tevatron Higgs hunt is on its way. The present situation of Higgs searches at the Tevatron is summarized in Fig. 11, showing some preliminary results 


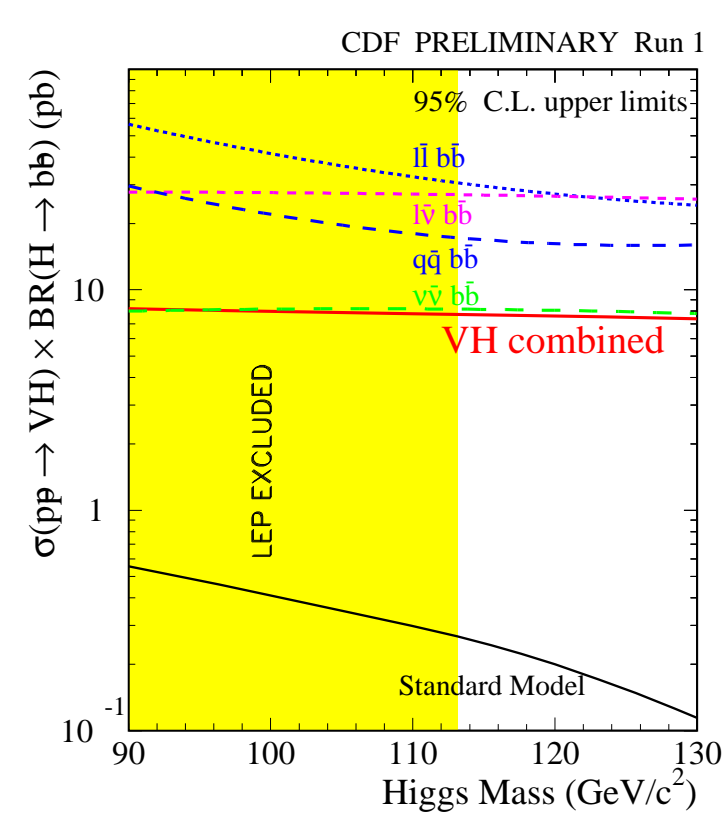

Figure 11. Preliminary upper limits (at 95\% c.l.) on production cross-sections times branching ratios, as functions of $m_{H}$, from Run I of the CDF experiment.

from CDF 4 (D0 had a slightly smaller sensitivity). With the luminosity and detectors of Run I, Tevatron is still more than one order of magnitude away from the sensitivity required by the SM Higgs properties. However, as described in detail in a dedicated study 50, and summarized in Fig. 12, things will be different, and very challenging, in the near future. For $m_{H}<135 \mathrm{GeV}$, the focus of the present attention, CDF and D0 will search for the SM Higgs boson considering its associated production with a weak gauge boson, $p \bar{p} \rightarrow V+(H \rightarrow b \bar{b})\left(V=W^{ \pm}, Z\right)$, and looking at a number of different final states: $(l \nu)(b \bar{b}),\left(l^{+} l^{-}\right)(b \bar{b}),(\nu \bar{\nu})(b \bar{b})$. Serious backgrounds are $V b \bar{b}, V V, t \bar{t}$, single top, and others. For $m_{H}>135 \mathrm{GeV}$, the channel $g g \rightarrow H \rightarrow W W^{(*)}$ becomes accessible, and the useful final states are $\left(l^{ \pm} l^{ \pm} j j\right)$ and $\left(l^{+} l^{-} \nu \bar{\nu}\right)$. (In this Section $l$ will always stand for $e$ or $\mu$.) The results of Fig. 12 are obtained by combining the statistical power of both experiments and all the channels mentioned

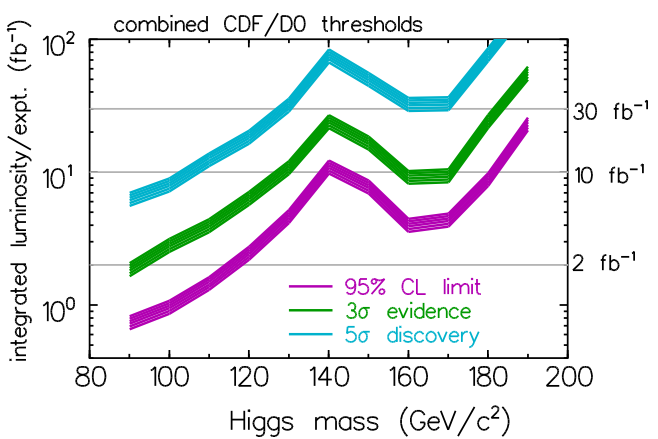

Figure 12. The integrated luminosity required per experiment, to either exclude a SM Higgs boson at $95 \%$ c.l. or discover it at the $3 \sigma$ or $5 \sigma$ level, as a function of $m_{H}$.

above. The lower edge of the bands is the calculated threshold; the bands extend upward from these nominal thresholds by $30 \%$ as an indication of the uncertainties in $b$ tagging efficiencies, background rate, mass resolution, and other effects.

The Higgs hunt will continue at the LHC (whose status is summarized by another talk at this Conference 34 ). In the mass region $m_{H}>130 \mathrm{GeV}$, the job of the ATLAS and CMS experiments will be relatively easy, thanks to the gold-plated channel $H \rightarrow Z Z^{(*)} \rightarrow 4 l^{ \pm}$, with other channels as a backup for the mass regions with less statistics: $H \rightarrow W W^{(*)} \rightarrow l \nu l \nu$ for $m_{H} \sim 2 m_{W} \pm 30 \mathrm{GeV}, H \rightarrow Z Z \rightarrow l^{+} l^{-} \nu \nu$ (and possibly $H \rightarrow W W \rightarrow l \nu j j$ or $H \rightarrow$ $\left.Z Z \rightarrow l^{+} l^{-} j j\right)$ for $m_{H}>600 \mathrm{GeV}$. In the case of a light Higgs, $m_{H}<130 \mathrm{GeV}$, various different signals are available. Earlier studies have defined the strategies for signals such as inclusive $H \rightarrow \gamma \gamma, t \bar{t}+(H \rightarrow b \bar{b}, \gamma \gamma)$ and $V+(H \rightarrow b \bar{b}, \gamma \gamma)$. The combined discovery potential of the ATLAS and CMS experiments, for different integrated luminosities, is summarized 51 in Fig. 13. During the last year, there was progress 22 in the study of the channel $q \bar{q} \rightarrow(W W \rightarrow H)+j j$ : exploiting the two tagged forward jets, the 


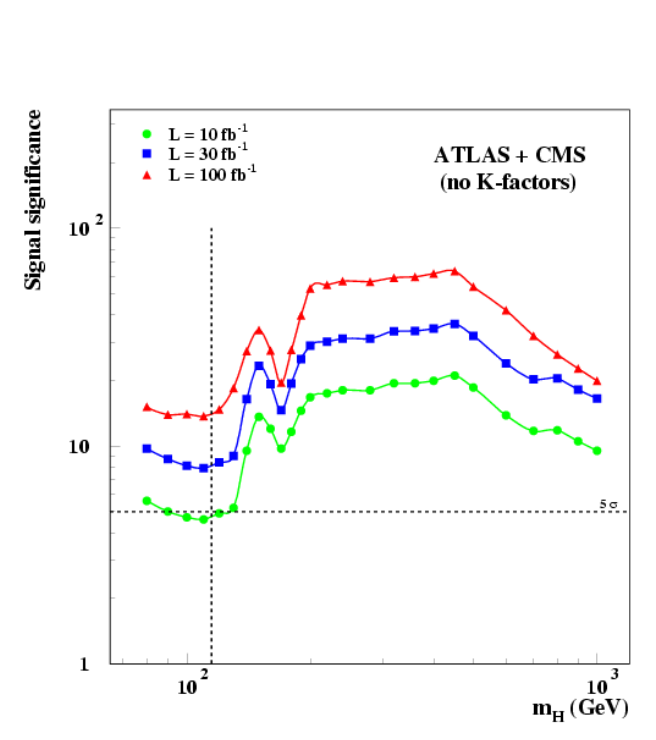

Figure 13. Sensitivity for the discovery of a SM Higgs boson at the LHC, as a function of $m_{H}$. The overall statistical significance, integrated over different channels, is plotted for three different integrated luminosities $\left(10,30\right.$ and $\left.100 \mathrm{fb}^{-1}\right)$, and assumes the combined statistical power of the ATLAS and CMS experiments.

background can be consistently reduced, allowing the study of decay channels such as $H \rightarrow W^{(*)} W^{*} \rightarrow l \nu l^{\prime} \nu$, which may be a discovery mode for $m_{H} \sim 115 \mathrm{GeV}$.

There are many other recent phenomenological studies on Higgs physics at highenergy colliders that would deserve a detailed discussion. Time limitations just permit a brief mention of some SM studies, with reference to the corresponding papers, leaving aside analogous studies for the MSSM and for more exotic possibilities. Soft and virtual NNLO QCD corrections to $g g \rightarrow H+$ $X$ have been computed 53. 'Strong' weak effects at high-energies (Bloch-Nordsieck violations) were studied 54: in particular, $\left(\alpha_{W} / \pi\right) \log ^{2}\left(s / m_{W}^{2}\right)$ corrections to $\sigma\left(e^{+} e^{-} \rightarrow\right.$ hadrons $)$ and an enhanced $m_{H}$ dependence in $W_{L} W_{L} \rightarrow$ hadrons. The crosssection for Higgs +2 jets via gluon-gluon fusion was computed 5. High- $p_{T}$ Higgs signals from $W W \rightarrow H \rightarrow b \bar{b}$ were investi- gated 56. The NLO QCD corrections to $p \bar{p}(p p) \rightarrow t \bar{t} H+X$ were computed by two different groups 5 .

\section{Conclusions}

In the presence of an experimental and theoretical puzzle, as recalled by the title assigned to this talk by the Organizers, conclusions can only be tentative.

It is clear that the search for the 'Higgs boson' (or, more generally, for the dynamics underlying the spontaneous breaking of the electroweak gauge symmetry) is the main goal of high-energy physics in the present decade.

Direct searches and electroweak precision tests strongly constrain the possibilities: with the presently available information, the existence of at least one light Higgs boson with SM-like (or MSSM-like) properties looks like the best bet, but there is still room for the unexpected.

It is important to stress that, in all 'natural' models, the Higgs boson is not alone: the accompanying physics may be even richer in implications (as, for example, in the case of supersymmetry), and we must be prepared to fully explore the $\mathrm{TeV}$ scale.

While not very successful so far, the theoretical search for plausible alternatives to the SM and the MSSM is worth pursuing, as confirmed by the many ongoing activities along different directions, in particular extra dimensions.

The final (scientific) judgement is coming, and experiment will express it with the help of run II of the Tevatron, of the LHC, and hopefully more facilities to come ...

In our quest for the fundamental laws of Nature, there is no substitute for the highenergy frontier! 
The Higgs Puzzle: Experiment and Theory [Lepton Photon 01, Rome, July 2001]

\section{Questions}

Q. Bennie Ward, MPI and Univ. of Tennessee:

There is the ultra-conservative view that we have a light Higgs, they will find it and there is nothing else. It is that way because God made it like that, unnatural or not. The theories in extra dimensions you mention are non-renormalizable so, if you would find them, you are still left with their non-renormalizable artifacts. Why would you say one of these scenarios is better than the other?

A. In view of naturalness arguments, the possibility of finding a light Higgs and nothing else at the weak scale seems unlikely. Rigorously, we cannot exclude that the gauge hierarchy problem is solved by mysterious infrared-ultraviolet connections that we are unable to understand with the tools of conventional quantum field theory. However, not even the cosmological constant violates so far the naturalness criterion, since gravitational interactions have been tested only up to energy scales of the order of $10^{-3} \mathrm{eV}$, not far from the phenomenological value of $\Lambda_{\text {cosm }}$ in the normalization of Eq. (13). Coming to the second part of your question, extra dimensions are just one out of many possibilities for the new physics at the Fermi scale. Their phenomenology may be described by an effective field theory, but the latter must eventually find an ultraviolet completion: this may be provided, for example, by an underlying superstring theory.

\section{Acknowledgments}

The author would like to thank Riccardo Paramatti and Paolo Valente for precious technical help in the preparation of the talk, Giuseppe Degrassi for discussions on the content of section 1.2, Juliet Lee-Franzini and
Pietro Slavich for useful comments. This work was supported in part by the European Union under the contracts HPRN-CT2000-00149 (Collider Physics) and HPRNCT-2000-00148 (Across the Energy Frontier).

\section{References}

1. P.W. Higgs, Phys. Lett. 12, 132 (1964); Phys. Rev. Lett. 13, 508 (1964); Phys. Rev. 145, 1156 (1966); F. Englert and R. Brout, Phys. Rev. Lett. 13, 321 (1964).

2. R. Barbieri, these Proceedings.

3. G.G. Hanson, these Proceedings.

4. The LEP Higgs Working Group, LHWG Note/2001-03, hep-ex/0107029.

5. J. Drees, these Proceedings.

6. A summary of the preliminary results of the LEP Electroweak Working Group is available at the LEPEWWG home page, http://lepewwg.web.cern.ch/LEPEWWG/

7. A. Freitas, W. Hollik, W. Walter and G. Weiglein, Phys. Lett. B 495, 338 (2000) hep-ph/0007091.

8. J. Z. Bai et al. [BES Collaboration], hep-ex/0102003; R. R. Akhmetshin et al. [CMD-2 Collaboration], hepex/9904027.

9. H. Burkhardt and B. Pietrzyk, Phys. Lett. B 513, 46 (2001).

10. A.D. Martin, J. Outhwaite and M.G. Ryskin, Eur. Phys. J. C 19, 681 (2001) hep-ph/0012231.

11. F. Jegerlehner, hep-ph/0104304.

12. L. Demortier, R. Hall, R. Hughes, B. Klima, R. Roser and M. Strovink [The Top Averaging Group for the CDF and D0 Collaborations], FERMILABTM-2084.

13. M.S. Chanowitz, hep-ph/0104024.

14. N. Cabibbo, L. Maiani, G. Parisi and R. Petronzio, Nucl. Phys. B 158, 295 (1979).

15. G. Isidori, G. Ridolfi and A. Strumia, Nucl. Phys. B 609, 387 (2001) hep- 
$\mathrm{ph} / 0104016]$.

16. C.D. Froggatt, H.B. Nielsen and Y. Takanishi, hep-ph/0104161.

17. J. Ellis, these Proceedings.

18. G. Altarelli, F. Caravaglios, G.F. Giudice, P. Gambino and G. Ridolfi, JHEP 0106, 018 (2001) hep-ph/0106029.

19. H. Georgi, H. R. Quinn and S. Weinberg, Phys. Rev. Lett. 33, 451 (1974); S. Dimopoulos, S. Raby and F. Wilczek, Phys. Rev. D 24, 1681 (1981); L. E. Ibanez and G. G. Ross, Phys. Lett. B 105, 439 (1981).

20. J. Ellis, G. Ridolfi and F. Zwirner, Phys. Lett. B 257, 83 (1991) and Phys. Lett. B 262, 477 (1991); Y. Okada, M. Yamaguchi and T. Yanagida, Prog. Theor. Phys. 85, 1 (1991) and Phys. Lett. B 262, 54 (1991); H. E. Haber and R. Hempfling, Phys. Rev. Lett. 66, 1815 (1991).

21. R. Hempfling and A.H. Hoang, Phys. Lett. $\quad$ B 331, 99 (1994) [hepph/9401219]; S. Heinemeyer, W. Hollik and G. Weiglein, Phys. Rev. D 58, 091701 (1998) hep-ph/9803277; Phys. Lett. B 440, 296 (1998) [hepph/9807423]; Eur. Phys. J. C 9, 343 (1999) hep-ph/9812472; Phys. Lett. B 455, 179 (1999) hep-ph/9903404; R. Zhang, Phys. Lett. B 447, 89 (1999) hep-ph/9808299; J.R. Espinosa and R. Zhang, JHEP 0003, 026 (2000) [hepph/9912236]; G. Degrassi, P. Slavich and F. Zwirner, Nucl. Phys. B 611, 403 (2001) hep-ph/0105096.

22. J.R. Espinosa and R. Zhang, Nucl. Phys. B 586, 3 (2000) thep$\mathrm{ph} / 0003246$.

23. A. Brignole, G. Degrassi, P. Slavich and F. Zwirner, preprint DFPD-01/TH/40, RM3-TH/01-14, ROME1-1327/01, to appear.

24. The LEP Higgs Working Group, LHWG Note/2001-04 hep-ex/0107030, LHWG Note/2001-05 hep-ex/0107031, LHWG
Note/2001-06 hep-ex/0107032 and LHWG Note/2001-08 hep-ex/0107035].

25. T. Affolder et al. [CDF Collaboration], Phys. Rev. Lett. 86, 4472 (2001) hep$\mathrm{ex} / 0010052$.

26. M. Carena, J.R. Ellis, A. Pilaftsis and C.E. Wagner, Phys. Lett. B 495, 155 (2000) [hep-ph/0009212].

27. S. Ambrosanio, A. Dedes, S. Heinemeyer, S. Su and G. Weiglein, hep$\mathrm{ph} / 0106255$.

28. T. Appelquist and C.W. Bernard, Phys. Rev. D 22, 200 (1980); A.C. Longhitano, Phys. Rev. D 22, 1166 (1980) and Nucl. Phys. B 188, 118 (1981).

29. B.A. Kniehl and A. Sirlin, Eur. Phys. J. C 16, 635 (2000) hep-ph/9907293; J.A. Bagger, A.F. Falk and M. Swartz, Phys. Rev. Lett. 84, 1385 (2000) hep$\mathrm{ph} / 9908327$.

30. W. Buchmuller and D. Wyler, Nucl. Phys. B 268, 621 (1986).

31. L.J. Hall and C. Kolda, Phys. Lett. B 459, 213 (1999) hep-ph/9904236; R. Barbieri and A. Strumia, Phys. Lett. B 462, 144 (1999) hep-ph/9905281; C. Kolda and H. Murayama, JHEP 0007, 035 (2000) hep-ph/0003170.

32. M.E. Peskin and J.D. Wells, Phys. Rev. D 64, 093003 (2001) hep-ph/0101342].

33. Y.Y. Kim, these Proceedings.

34. L. Maiani, these Proceedings.

35. R. Heuer, these Proceedings.

36. M. Turner, these Proceedings.

37. L. Randall, these Proceedings.

38. I. Antoniadis and K. Benakli, Int. J. Mod. Phys. A 15, 4237 (2000) hep-ph/0007226; V. A. Rubakov, hep$\mathrm{ph} / 0104152$.

39. R. Rohm, Nucl. Phys. B 237, 553 (1984); C. Kounnas and M. Porrati, Nucl. Phys. B 310, 355 (1988); S. Ferrara, C. Kounnas, M. Porrati and F. Zwirner, Nucl. Phys. B 318, 75 (1989).

40. I. Antoniadis, Phys. Lett. B 246, 377 
(1990).

41. J. Scherk and J. H. Schwarz, Phys. Lett. B 82, 60 (1979) and Nucl. Phys. B 153, 61 (1979); P. Fayet, Phys. Lett. B 159, 121 (1985) and Nucl. Phys. B 263, 649 (1986).

42. I. Antoniadis, S. Dimopoulos and G. R. Dvali, Nucl. Phys. B 516, 70 (1998) hep-ph/9710204.

43. A. Delgado, A. Pomarol and M. Quiros, Phys. Rev. D 60, 095008 (1999) hep-ph/9812489; I. Antoniadis, K. Benakli and M. Quiros, hep-th/0108005; R. Barbieri, L.J. Hall and Y. Nomura, Phys. Rev. D 63, 105007 (2001) hep-ph/0011311; N. Arkani-Hamed, L.J. Hall, Y. Nomura, D.R. Smith and N. Weiner, Nucl. Phys. B 605, 81 (2001) hep-ph/0102090; G.R. Dvali, S. Randjbar-Daemi and R. Tabbash, hep-ph/0102307.

44. H. Georgi, A. K. Grant and G. Hailu, Phys. Lett. B 506, 207 (2001) hep-ph/0012379; R. Contino, L. Pilo, R. Rattazzi and A. Strumia, JHEP 0106, 005 (2001) hep-ph/0103104; N. Arkani-Hamed, A. G. Cohen and H. Georgi, Phys. Lett. B 516, 395 (2001) hep-th/0103135. For some works appeared after the Conference, see also: D. M. Ghilencea, S. Groot Nibbelink and H. P. Nilles, hep-th/0108184; C. A. Scrucca, M. Serone, L. Silvestrini and F. Zwirner, hep-th/0110073.

45. C. Kounnas, F. Zwirner and I. Pavel, Phys. Lett. B 335, 403 (1994) hep$\mathrm{ph} / 9406256$.

46. W.D. Goldberger and M.B. Wise, Phys. Rev. Lett. $\quad \mathbf{8 3}, 4922$ (1999) [hep$\mathrm{ph} / 9907447$.

47. N.

Arkani-Hamed, M. Porrati and L. Randall, JHEP 0108, 017 (2001) hep-th/0012148; R. Rattazzi and A. Zaffaroni, JHEP 0104, 021 (2001) hep-th/0012248.

48. G.F. Giudice, R. Rat- tazzi and J.D. Wells, Nucl. Phys. B 595, 250 (2001) hep-ph/0002178; E. Perazzi, G. Ridolfi and F. Zwirner, Nucl. Phys. B 574, 3 (2000) hep-ph/0001025 and B 590, 287 (2000) hep-ph/0005076.

49. L. Moneta [CDF collaboration], hepex/0106050.

50. M. Carena et al., hep-ph/0010338.

51. F. Gianotti, based on the ATLAS Technical Design Report, CERN/LHCC/9914. For a recent review, appeared after this Conference, see also: J. G. Branson, D. Denegri, I. Hinchliffe, F. Gianotti, F. E. Paige and P. Sphicas (for the ATLAS and CMS Collaborations), hep$\mathrm{ph} / 0110021$.

52. N. Kauer, T. Plehn, D. Rainwater and D. Zeppenfeld, Phys. Lett. B 503, 113 (2001) hep-ph/0012351.

53. S. Catani, D. de Florian and M. Grazzini, JHEP 0105, 025 (2001) hep-ph/0102227; R.V. Harlander and W.B. Kilgore, Phys. Rev. D 64, 013015 (2001) hep-ph/0102241.

54. M. Ciafaloni, P. Ciafaloni and D. Comelli, Phys. Rev. Lett. 87, 211802 (2001) hep-ph/0103315 and Nucl. Phys. B 613, 382 (2001) hepph/0103316.

55. V. Del Duca, W. Kilgore, C. Oleari, C. Schmidt and D. Zeppenfeld, Phys. Rev. Lett. 87, 122001 (2001) hep$\mathrm{ph} / 0105129$.

56. V.A. Khoze, A.D. Martin and M.G. Ryskin, Eur. Phys. J. C 21, 99 (2001) [hep-ph/0104230.

57. W. Beenakker, S. Dittmaier, M. Kramer, B. Plumper, M. Spira and P.M. Zerwas, Phys. Rev. Lett. 87, 201805 (2001) hep-ph/0107081; L. Reina and S. Dawson, Phys. Rev. Lett. 87, 201804 (2001) hep-ph/0107101. 